\title{
Effects of Different Fertilization Treatments on Soil, Leaf Nutrient and Fruit Quality of Citrus grandis var. longanyou*
}

\author{
Rongfei Li', Yaodong Chang1, Tao Hu${ }^{2}$, Xueyou Jiang², Guolu Liang1, Zhiming Lu', Youwen $\mathrm{Yi}^{3}$, \\ Qigao Guo'"\#
}

${ }^{1}$ College of Horticulture and Landscape Architecture, Southwest University, Beibei Chongqing 400716, China

${ }^{2}$ Guang'an District Agricultural Bureau, Guang'an Sichuan, 638500, China

${ }^{3}$ Guang'an Science and Technology Development and Training Center, Guang'an Sichuan, 638500, China

Email:"qgguo@126.com

How to cite this paper: Li, R.F., Chang, Y.D., Hu, T., Jiang, X.Y., Liang, G.L., Lu, Z.M., Yi, Y.W. and Guo, Q.G. (2017) Effects of Different Fertilization Treatments on Soil, Leaf Nutrient and Fruit Quality of Citrus grandis var. longanyou. World Journal of Engineering and Technology, 5, 1-14.

https://doi.org/10.4236/wjet.2017.52B001

Received: March 7, 2017

Accepted: May 8, 2017

Published: May 11, 2017

\begin{abstract}
The five-year-old "Longanyou" trees were used as the experimental material to study the effects of different fertilization treatments. The nutrient contents in soil and leaves, fruit yield and quality were determined, and then the correlations were analyzed. The results showed that: 1) The soil nutrient contents of $0-20 \mathrm{~cm}$ depth were more than the $20-40 \mathrm{~cm}$, and the trends of nutrient contents of the $0-20 \mathrm{~cm}$ soil layers were as follows: treatment 2 (T2) $>$ treatment $3(\mathrm{~T} 3)>$ treatment $4(\mathrm{~T} 4)>$ treatment $1(\mathrm{~T} 1)>$ control $(\mathrm{CK})$. However, the $20-40 \mathrm{~cm}$ depth had not significant difference between different treatments, but T2, T4 and T3 were higher than T1 and CK. It indicated that the soil effective nutrient content increased in T2 and T3. 2) Compared with the control, the content of $\mathrm{K}$ and $\mathrm{B}$ elements was improved obviously in leaves with the increase of organic manure application. The contents of $\mathrm{P}$ (1.60 $\left.\mathrm{g} \cdot \mathrm{kg}^{-1}\right), \mathrm{B}\left(26.00 \mathrm{mg} \cdot \mathrm{kg}^{-1}\right)$ and $\mathrm{Mg}\left(1.18 \mathrm{~g} \cdot \mathrm{kg}^{-1}\right)$ were the highest, and other nutrients contents were also higher, indicating that T2 could effectively improve the leaves' nutrient contents. 3) The fruit yield per plant was the highest in T2 (95.40 kg plant ${ }^{-1}$ ), and the single fruit weight, total sugar, sugar and acid ratio, vitamin $\mathrm{C}$ were also the highest, but titratable acid was lower. It indicated that T2 effectively improved fruit yield and quality. 4) There were positive correlations between multiple factors of soil nutrients and the quality index, such as fruit peel thickness, total sugar, solid acid ratio, sugar and acid ratio, $\mathrm{Vc}$ content and single yield etc. There was significant correlation between $\mathrm{K}, \mathrm{B}, \mathrm{Zn}, \mathrm{Fe}$ contents and fruit yield and quality index, and the contents of $\mathrm{B}, \mathrm{Zn}$ and $\mathrm{Fe}$ in leaves were significantly correlated with soil nutrient, indicating that the contents of $\mathrm{K}, \mathrm{B}, \mathrm{Zn}, \mathrm{Fe}$ in soil and leaf were closely related to fruit yield and quality. In sum, the T2 was the best fertilization scheme for orchard management practice of "Longanyou".
\end{abstract}

${ }^{\star}$ Funded projects: National science \& technology support projects (2014BAD16B0102-I). 


\section{Keywords}

Citrus grandis var. longanyou, Fertilization, Soil Nutrient, Leaf Nutrient, Fruit Quality

\section{Introduction}

Soil is a fundamental factor of fruit production, and fruit trees usually absorb water and nutrients by their roots, therefore fertilizers are traditionally applied into the soil. The soil nutrient elements will directly affect on growth, fruit set, retention, yield and quality improvement and the sustainable production of orchard [1]. So the efficient use of fertilizers to increase fruit yield is an important goal in all agricultural manipulation systems. However, soil fertilization also causes environmental contamination for nutrients leaching into ground water [2], thus improving soil physical and chemical properties by the rational fertilization tillage is the efficient way to reduce the environmental pollution and increase the capacity of soil fixation and tree absorption. Moreover, plant leaves extremely sensitive respond to the soil mineral nutrient, and to a certain extent, the content of mineral nutrient in leaves can reflect the nutrient status of the trees and soil [3]. Therefore, soil and leaf nutrient have a significant effect on fruit yield and quality, and especially, the index of the content of leaf nutrient can respond to the nutritional requirements more accurately for optimum fruit yield [4]. That is, soil nutrient provides the necessary mineral elements for plant growth, and different fertilization treatments influence on the level of leaf nutrients [5], and mineral nutrition is the material basis of fruit tree growth and development, yield formation and fruit quality improvement, so the leaf nutrient is closely related to fruit quality [6] [7]. The correlation between the soil and leaf nutrient content and fruit yield and quality, was mainly studied on apple [8], pear [9], navel orange [6] and so on, but there has not yet reached a uniform conclusion about the correlation. Therefore, continuing to investigate the correlation, especially focusing on improving soil physical and chemical properties, nutrition level of tree and fruit yield and quality in the different fertilization treatments, is the long-term and beneficial way to fruit production.

Citrus grandis var. longanyou is a special fruit tree in Guang'an City, Sichuan Province. It was approved as a national geographical indication protection product in 2008 [10] [11]. However, abandoned cultivation is widespread in farmers' orchard management practices; especially in hilly land zone of Guang'an, the soil fertility conditions are different and generally undesirable, which result in low nutrient flowing in tree and consequently cultivar reproductive potentials do not become evident, and eventually lead to low yield, poor quality and poor economic value. The main aim of this study was to investigate the effects of different fertilization treatments on nutrient and fruit quality of "Longanyou", and then analyze the correlation, which will directly provide the beneficial reference 
for "Longanyou" fertilization management.

\section{Materials and Methods}

\subsection{Plant Growth Environment}

The experiment was proceed in Long'an, a township of Guang'an City during 2015-2016. The average elevation is $320 \mathrm{~m}$, the annual average temperature is $17.58^{\circ} \mathrm{C}$. The average temperature is $4^{\circ} \mathrm{C}$ in January, it is the coldest in a year. The average temperature is $34^{\circ} \mathrm{C}$ in July, it is the hottest in a year. Frost-free has $306-328 \mathrm{~d}$, the average annual rainfall of $1240 \mathrm{~mm}$, the average annual sunshine hours of $1213 \mathrm{~h}$. The testing area which is a typical southwest hilly region of high temperature and rainy climate, and soil fertility is low.

\subsection{Experimental Design}

The experiments were conducted in a 5-year-old plantation, with a $5.0 \mathrm{~m} \times 5.3$ $\mathrm{m}$ planting spacing. In the experiment, 5 fertilizer schemes were set up. The treatments were as follows: treatment 1 (T1: nitrogen $315 \mathrm{~kg}$, phosphorus 173.25 $\mathrm{kg}$, potassium $306 \mathrm{~kg}$ and organic matter $1575 \mathrm{~kg}$ were used per hectare), treatment 2 (T2: nitrogen $450 \mathrm{~kg}$, phosphorus $247.5 \mathrm{~kg}$, potassium $438 \mathrm{~kg}$ and organic matter $2250 \mathrm{~kg}$ were used in per hectare), treatment 3 (T3: all applied organic fertilizer, $3000 \mathrm{~kg}$ in per hectare), treatment 4 (T4: total soluble fertilizer was reduced with fertilization times increasing, nitrogen $202.8 \mathrm{~kg}$, phosphorus $252.3 \mathrm{~kg}$ and potassium $268.95 \mathrm{~kg}$ were used in per hectare), and control (CK: the trees that abandoned by farmers in the production). The applying fertilizer method: open hole $(0.4 \mathrm{~m} \times 0.4 \mathrm{~m})$. The fertilization time is divided into: Basal fertilizer (November 10-15, 2015), Sprout fertilizer (February 25-30, 2016), and strong fruit fertilizer (May 25-30, 2016). One tree as a plot, each treatment has 15 trees.

\subsection{Methods}

\subsubsection{Soil Sampling and Nutrient Elements Determination}

Soil sampling and determination were done following the protocols of as described by Tang et al. [12] and Zhang et al. [13]. In each treatment 15 - 20 sampling tree were chosen by "S" curve. A $200 \mathrm{~g}$ soil samples was collected under each tree crown at a $0-20 \mathrm{~cm}$ and $20-40 \mathrm{~cm}$ depth respectively, then mixed soil samples collected from 15 - 20 sampling sites and a $500 \mathrm{~g}$ soil was selected using a diagonal sampling method.

Soil chemical properties were analyzed by traditional analytical methods as described by Bao [14]. $\mathrm{pH}$ of soil was determined by potentiometrically in 1:1 soil/ distilled water suspensions after shaking. Organic matter content was determined by $\mathrm{K}_{2} \mathrm{Cr}_{2} \mathrm{O}_{7}$ oxidation external heating method. Total nitrogen (N) was determined by Semi-microkine Kjeldahl method, and alkaline $\mathrm{N}$ was determined by alkali hydrolysis diffusion method. Total phosphorus $(\mathrm{P})$ was determined by $\mathrm{HCIO}_{4}-\mathrm{H}_{2} \mathrm{SO}_{4}$ decomposition and Molybdenum antimony anti-colorimetric method. Total potassium $(\mathrm{K})$ content was determined by $\mathrm{HF}-\mathrm{HClO}_{4}$ decomposition and atomic absorption method. The available $\mathrm{P}$ content was extracted by 
$\mathrm{NaHCO}_{3}$ solution, Colorimetric method. The available $\mathrm{K}$ content determination uses ammonium acetate solution extraction, atomic absorption method [15]. The effective copper $(\mathrm{Cu})$, iron (Fe), manganese $(\mathrm{Mn})$, Zinc $(\mathrm{Zn})$ and exchangeable calcium ( $\mathrm{Ca}$ ) and magnesium $(\mathrm{Mg})$ were determined by atomic absorption spectrophotometer. The effective boron (B) was determined by boiling water extraction-ICP-AES method [12].

\subsubsection{Leaf Sampling and Nutrient Elements Determination}

Leaves samples were collected from four points of the half height canopy of the test trees, which located in north, south, east and west of the test trees. 15 healthy and mature leaves were respectively collected from the vegetative branches and bearing branches of each testing tree which have the same growth vigor, the samples were transported to the lab on ice in a cooler and stored in an icebox for elements determination. Their elements were determined following the protocols of as described by Zhuang et al. [16] and Diao [17]. The N content was analyzed by Kay type nitrogen method. The P content was determined by molybdenum antimony anti-color method. The contents of $\mathrm{K}, \mathrm{Mg}, \mathrm{Fe}$ and $\mathrm{Zn}$ were determined by atomic absorption spectrometry. The content of $B$ was determined by dry ashing-imine colorimetric method.

\subsubsection{Fruit Sampling and Yield, Quality Determination}

Fruit yield of per plant and per hectare in different fertilization treatments were tested. And then respectively one fruit located in north, south, east, west and center of upper and lower canopy of each test tree were collected and transported to the lab. Their appearance quality was measured after washing and drying, fruit juice used as internal quality sample. Repeat 5 times.

The Total Soluble Solids (TSS), Titratable Acidity (TA), Vitamin C (Vc content), Total Sugar, Sucrose, Invert sugar and Reducing Sugar were measured according to national standard GB8210-87. And calculating

The ratio of solid and acid = soluble solids/titratable acid,

Sugar and acid ratio = total sugar/titratable acid.

Per fruit weight, fruit thickness, vertical diameter and transverse diameter of fruit were measured with a vermier caliper, and calculating

The fruit shape index $=$ vertical diameter/transverse diameter.

\subsection{Statistical Analysis}

Data sorting with Excel, SPSS13.0 software was used for analysis of variance and correlation analysis.

\section{Results}

\subsection{Soil Nutrient Contents}

The effects of different fertilization treatments on the nutrient contents in different soil layers were inconsistent. Among them, the trend of nutrient content of 0 $-20 \mathrm{~cm}$ soil layers were as follows: $\mathrm{T} 2>\mathrm{T} 3>\mathrm{T} 4>\mathrm{T} 1>\mathrm{CK}$. The treatments of 20 
- $40 \mathrm{~cm}$ soil layers were not significant, but T2, T4 and T3 were higher than those of T1 and CK. The different soil layers in same treatment showed: $0-20 \mathrm{~cm}>20$ - $40 \mathrm{~cm}$ soil layer (Table 1). But the contents of total $\mathrm{N}$, alkaline $\mathrm{N}$, available $\mathrm{Fe}$,Vailable $\mathrm{Mn}$ and Exchangeable $\mathrm{Mg}$ were the highest in $0-20 \mathrm{~cm}$ soil layer of T3, the contents were: $1.49 \mathrm{mg} \cdot \mathrm{kg}^{-1}, 138.00 \mathrm{mg} \cdot \mathrm{kg}^{-1}, 56.74 \mathrm{mg} \cdot \mathrm{kg}^{-1}, 6.75 \mathrm{mg} \cdot \mathrm{kg}^{-1}, 0.62$ $\mathrm{mg} \cdot \mathrm{kg}^{-1}$, respectively. And the organic matter content of T3 $\left(17.2 \mathrm{~g} \cdot \mathrm{kg}^{-1}\right)$ was significantly higher than that of T4, T1, CK and $20-40 \mathrm{~cm}$ soil layer of T2. Compared with the control, the organic matter, total $\mathrm{P}$, alkaline $\mathrm{N}$, available $\mathrm{P}$, available $\mathrm{K}$, available $\mathrm{B}$, available $\mathrm{Cu}$, available $\mathrm{Zn}$ and available $\mathrm{Mn}$ were significantly improved in $0-20 \mathrm{~cm}$ soil layer. Then T2 and T3 were beneficial to promote soil fertility levels.

\subsection{Leaf Nutrient Contents}

The different fertilization treatments had significant differences in the leaves' nutrient content of "Longanyou" (Table 2). Compared with the control, the

Table 1. The soil nutrient contents of different fertilization treatments in Longanyou orchard.

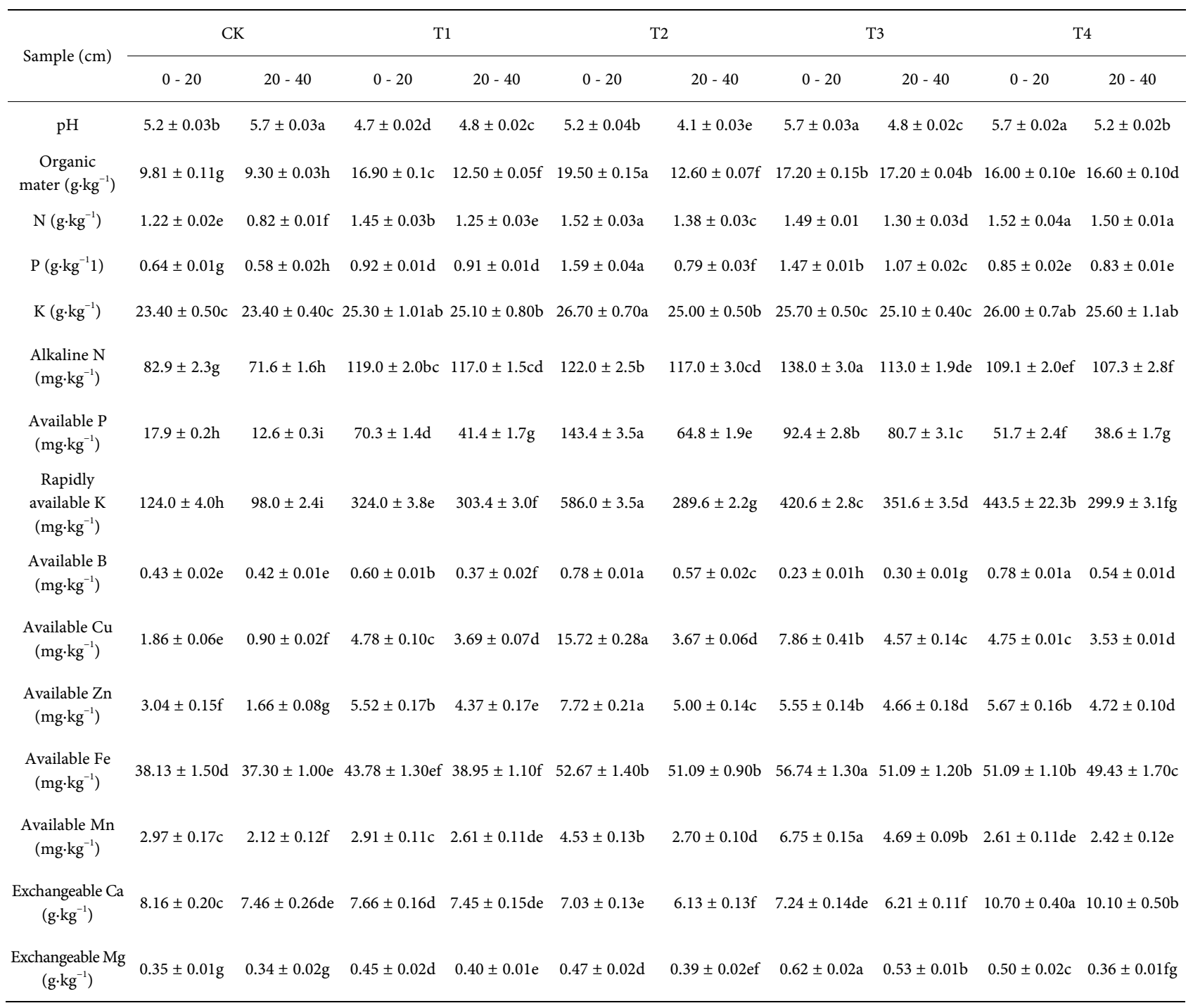

Note: Values within a column followed by the different lowercase letter are significantly different at 0.05 level. 
Table 2. The leaves' nutrient content of different fertilization treatments in Longanyou.

\begin{tabular}{ccccccccc}
\hline Treatment & Branch type & $\mathrm{N}\left(\mathrm{g} \cdot \mathrm{kg}^{-1}\right)$ & $\mathrm{P}\left(\mathrm{g} \cdot \mathrm{kg}^{-1}\right)$ & $\mathrm{K}\left(\mathrm{g} \cdot \mathrm{kg}^{-1}\right)$ & $\mathrm{B}\left(\mathrm{mg} \cdot \mathrm{kg}^{-1}\right)$ & $\mathrm{Mg}\left(\mathrm{g} \cdot \mathrm{kg}^{-1}\right)$ & $\mathrm{Zn}\left(\mathrm{mg} \cdot \mathrm{kg}^{-1}\right)$ & $\mathrm{Fe}\left(\mathrm{mg} \cdot \mathrm{kg}^{-1}\right)$ \\
\hline \multirow{2}{*}{$\mathrm{CK}$} & Bearing branch & $24.00 \pm 0.10 \mathrm{~d}$ & $1.13 \pm 0.02 \mathrm{de}$ & $8.33 \pm 0.07 \mathrm{f}$ & $11.47 \pm 0.07 \mathrm{e}$ & $0.85 \pm 0.01 \mathrm{ef}$ & $8.39 \pm 0.07 \mathrm{f}$ & $72.80 \pm 0.10 \mathrm{~d}$ \\
& Vegetative branch & $24.20 \pm 0.10 \mathrm{~d}$ & $1.16 \pm 0.04 \mathrm{~d}$ & $10.80 \pm 0.05 \mathrm{~d}$ & $11.77 \pm 0.53 \mathrm{e}$ & $0.96 \pm 0.01 \mathrm{c}$ & $9.20 \pm 0.03 \mathrm{e}$ & $69.70 \pm 0.10 \mathrm{ef}$ \\
$\mathrm{T} 1$ & Bearing branch & $23.00 \pm 0.44 \mathrm{e}$ & $1.03 \pm 0.03 \mathrm{f}$ & $10.06 \pm 0.12 \mathrm{e}$ & $16.93 \pm 0.75 \mathrm{~d}$ & $0.88 \pm 0.03 \mathrm{de}$ & $8.40 \pm 0.24 \mathrm{f}$ & $71.27 \pm 1.06 \mathrm{de}$ \\
& Vegetative branch & $27.00 \pm 0.51 \mathrm{a}$ & $1.32 \pm 0.08 \mathrm{c}$ & $10.63 \pm 0.15 \mathrm{de}$ & $17.20 \pm 0.26 \mathrm{~d}$ & $1.10 \pm 0.02 \mathrm{~b}$ & $10.20 \pm 0.34 \mathrm{bc}$ & $69.13 \pm 1.14 \mathrm{f}$ \\
$\mathrm{T} 2$ & Bearing branch & $23.83 \pm 0.80 \mathrm{~d}$ & $1.29 \pm 0.06 \mathrm{c}$ & $11.63 \pm 0.30 \mathrm{bc}$ & $25.40 \pm 1.65 \mathrm{a}$ & $0.80 \pm 0.02 \mathrm{f}$ & $9.75 \pm 0.19 \mathrm{~cd}$ & $95.80 \pm 2.00 \mathrm{a}$ \\
& Vegetative branch & $27.97 \pm 0.20 \mathrm{~b}$ & $1.60 \pm 0.04 \mathrm{a}$ & $12.23 \pm 0.76 \mathrm{ab}$ & $26.00 \pm 0.36 \mathrm{a}$ & $1.18 \pm 0.05 \mathrm{a}$ & $10.31 \pm 0.42 \mathrm{~b}$ & $79.37 \pm 1.02 \mathrm{c}$ \\
\multirow{2}{*}{ T3 } & Bearing branch & $23.60 \pm 0.30 \mathrm{de}$ & $1.15 \pm 0.01 \mathrm{~d}$ & $10.03 \pm 0.08 \mathrm{e}$ & $18.70 \pm 0.20 \mathrm{c}$ & $0.94 \pm 0.03 \mathrm{c}$ & $11.25 \pm 0.05 \mathrm{a}$ & $69.25 \pm 0.95 \mathrm{ef}$ \\
& Vegetative branch & $25.95 \pm 0.15 \mathrm{c}$ & $1.26 \pm 0.02 \mathrm{c}$ & $11.44 \pm 0.24 \mathrm{c}$ & $20.20 \pm 0.20 \mathrm{~b}$ & $1.11 \pm 0.03 \mathrm{~b}$ & $11.30 \pm 0.40 \mathrm{a}$ & $69.85 \pm 0.75 \mathrm{ef}$ \\
\multirow{2}{*}{ T4 } & Bearing branch & $26.60 \pm 0.46 \mathrm{bc}$ & $1.08 \pm 0.03 \mathrm{ef}$ & $12.13 \pm 0.61 \mathrm{ab}$ & $18.73 \pm 0.31 \mathrm{c}$ & $0.93 \pm 0.04 \mathrm{~cd}$ & $9.41 \pm 0.25 \mathrm{de}$ & $81.27 \pm 0.81 \mathrm{~b}$ \\
& Vegetative branch & $28.10 \pm 0.53 \mathrm{a}$ & $1.48 \pm 0.02 \mathrm{~b}$ & $12.57 \pm 0.38 \mathrm{a}$ & $19.70 \pm 0.44 \mathrm{bc}$ & $1.11 \pm 0.04 \mathrm{~b}$ & $10.27 \pm 0.31 \mathrm{~b}$ & $69.87 \pm 1.45 \mathrm{ef}$ \\
\hline
\end{tabular}

Note: Values within a column followed by the different lowercase letter are significantly different at 0.05 level. The same as followed.

content of $\mathrm{K}$ and $\mathrm{B}$ increased significantly with the increase of organic manure application, but had little effect on the content of $\mathrm{P}$ in leaf. The $\mathrm{Zn}$ content in the vegetative branches and bearing branches of $\mathrm{T} 3$ were higher. The content of $\mathrm{P}$ $\left(1.60 \mathrm{~g} \cdot \mathrm{kg}^{-1}\right), \mathrm{B}\left(26.00 \mathrm{mg} \cdot \mathrm{kg}^{-1}\right)$ and $\mathrm{Mg}\left(1.18 \mathrm{~g} \cdot \mathrm{kg}^{-1}\right)$ were the highest, and $\mathrm{N}, \mathrm{K}$, $\mathrm{Zn}$ and Fe content were relatively higher than others. While the nutrient contents of control leaves' were the lowest. The nutrient contents of different branches were different, the trend was vegetative branches higher than bearing branches, but the Fe content was the opposite. Compared comprehensively, the nutrient contents of leaves in $\mathrm{T} 2$ were higher than others.

\subsection{Fruit Yield and Quality}

According to Figure 1(a), the yield per plant of T2 was the highest, $95.40 \mathrm{~kg}$ plant $^{-1}$, followed by T4, and the lowest was control, only $14.13 \mathrm{~kg}$ plant $^{-1}$. The fruit number per tree of $\mathrm{T} 3$ was the highest, followed by $\mathrm{T} 2$, and the lowest was the control (Figure 1(b)).

According to the comparison of the appearance quality of the fruit (Table 3), There were significant difference in the average fruit weight among the treatments. The fruit weight of the upper layer was the largest $(1736.70 \mathrm{~g})$ in T2, and it was 2.01 and 2.58 times of the upper and lower in control, respectively. Then followed by T4 upper layer $(1662.2 \mathrm{~g})$. Moreover, the fruit peel of T2 and T4 were thicker than T1, T3 and CK, which indicating that T2 and T4 can improve the ability of anti-fungal invasion and enhance the storability, combining with the fruit weight, we suggested that $\mathrm{T} 2$ and $\mathrm{T} 4$ were benefit for increasing the production and commodity value of Longanyou.

It was observed in Table 4 that the internal quality including the converting sugar, the total sugar, the ratio of sugar to acid, and the content of $\mathrm{Vc}$ were the highest in the upper layer fruits of the T2, followed by the upper layer fruits of T3. Titratable acid of the upper layer fruits were lower in T2 and T3, it was $0.66 \%$ and $067 \%$ respectively. Soluble solids (12.70\%), solid acid ratio (19.01), 
Fruit yield $\left(\mathrm{kg} \mathrm{plant}^{-1}\right)$

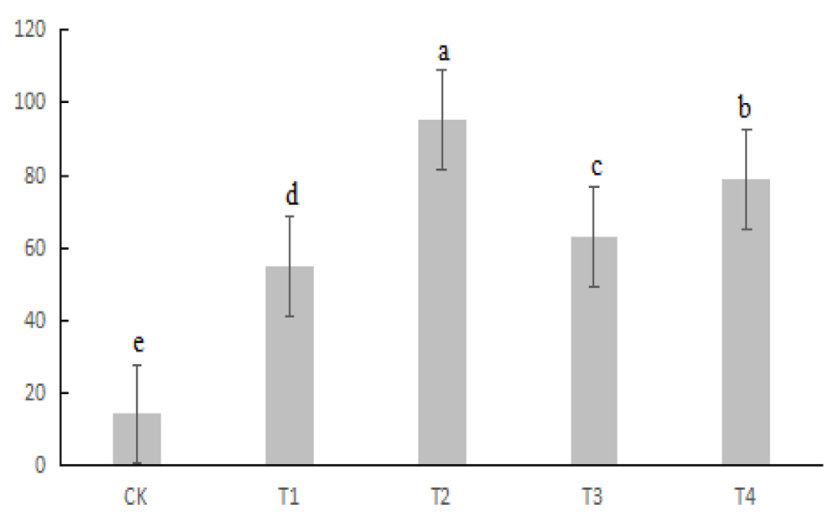

(a)
Fruit number (n plant $\mathrm{t}^{-1}$ )

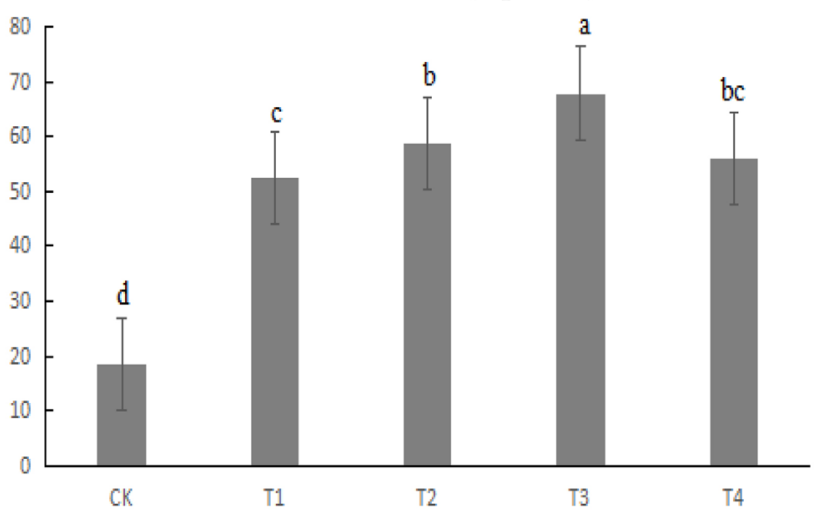

(b)

Figure 1. Fruit yield (a) and number (b) in different fertilization treatments.

Table 3. The fruits appearance quality of different fertilization treatments in Longanyou.

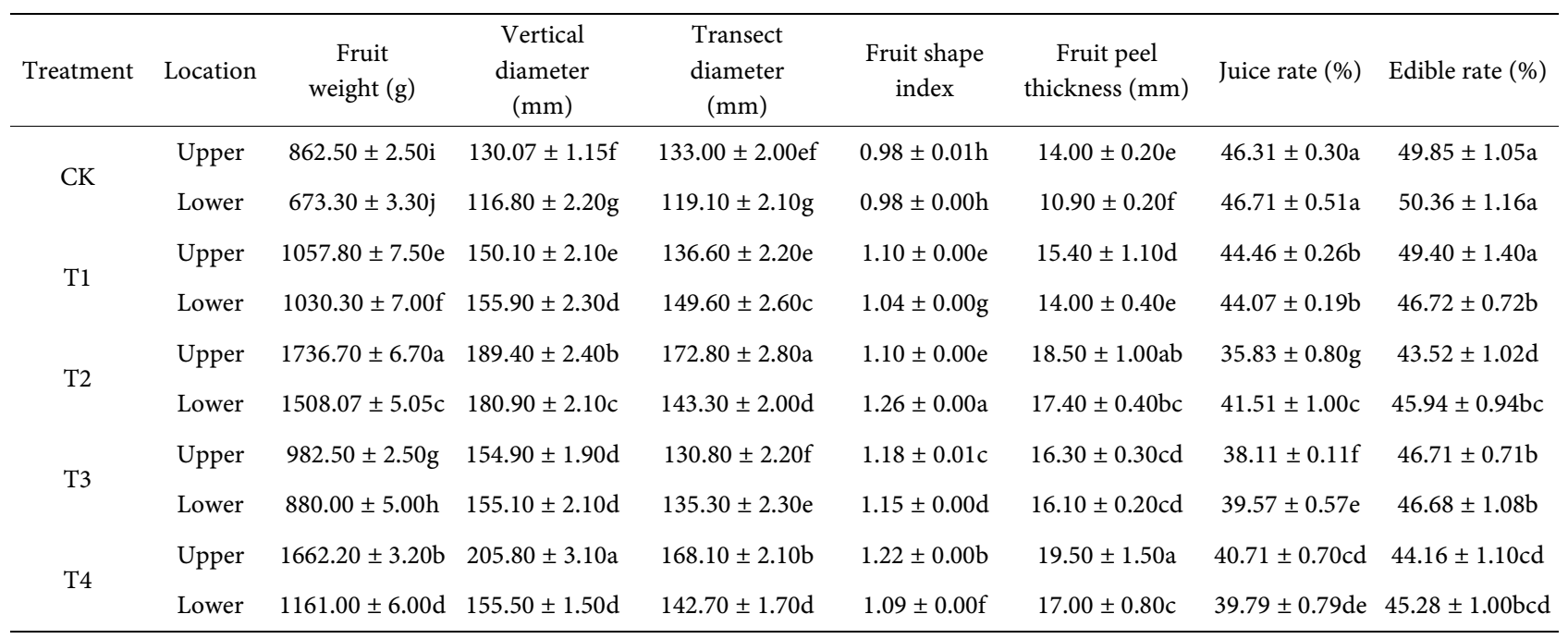

Table 4. The fruit internal quality of different fertilization treatments in Longanyou.

\begin{tabular}{|c|c|c|c|c|c|c|c|c|c|c|}
\hline 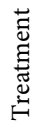 & 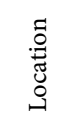 & $\underset{\mathscr{b}}{\stackrel{Ð}{\infty}}$ & 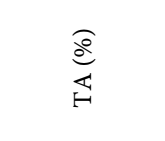 & 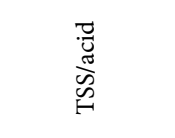 & 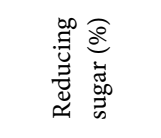 & 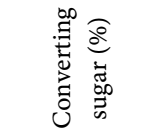 & 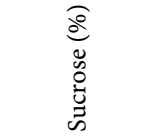 & 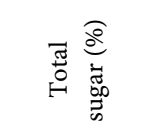 & 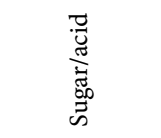 & 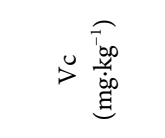 \\
\hline \multirow{2}{*}{ CK } & Upper & $10.30 \pm 0.30 \mathrm{e}$ & $0.79 \pm 0.01 b$ & $13.07 \pm 0.47 \mathrm{~d}$ & $3.23 \pm 0.13 b$ & $7.26 \pm 0.16 f$ & $3.62 \pm 0.22 \mathrm{~d}$ & $7.07 \pm 0.07 f$ & $8.97 \pm 0.16 \mathrm{e}$ & $53.89 \pm 1.34 \mathrm{~b}$ \\
\hline & Lower & $9.30 \pm 0.10 \mathrm{f}$ & $0.86 \pm 0.00 \mathrm{a}$ & $10.82 \pm 0.14 \mathrm{e}$ & $3.41 \pm 0.11 b$ & $6.81 \pm 0.11 \mathrm{~g}$ & $2.41 \pm 0.10 \mathrm{e}$ & $6.68 \pm 0.10 \mathrm{~g}$ & $7.78 \pm 0.10 f$ & $50.10 \pm 1.05 c$ \\
\hline \multirow{2}{*}{$\mathrm{T} 1$} & Upper & $10.60 \pm 0.30 \mathrm{de}$ & $0.69 \pm 0.00 \mathrm{de}$ & $15.28 \pm 0.45 b c$ & $3.75 \pm 0.15 \mathrm{ab}$ & $8.07 \pm 0.07 d$ & $4.56 \pm 0.11 b c$ & $7.96 \pm 0.16 \mathrm{~cd}$ & $11.47 \pm 0.20 c$ & $54.42 \pm 1.09 \mathrm{~b}$ \\
\hline & Lower & $10.30 \pm 0.30 \mathrm{e}$ & $0.78 \pm 0.04 \mathrm{bc}$ & $13.31 \pm 1.00 \mathrm{~d}$ & $3.71 \pm 0.11 \mathrm{ab}$ & $8.20 \pm 0.10 \mathrm{~cd}$ & $4.40 \pm 0.14 c$ & $7.83 \pm 0.13 d$ & $10.11 \pm 0.34 \mathrm{~d}$ & $52.43 \pm 1.26 \mathrm{bc}$ \\
\hline \multirow{2}{*}{$\mathrm{T} 2$} & Upper & $12.00 \pm 0.50 \mathrm{~b}$ & $0.66 \pm 0.01 \mathrm{f}$ & $18.29 \pm 0.81 \mathrm{a}$ & $4.51 \pm 0.11 \mathrm{ab}$ & $9.89 \pm 0.19 a$ & $4.60 \pm 0.10 \mathrm{bc}$ & $9.62 \pm 0.22 a$ & $14.66 \pm 0.34 \mathrm{a}$ & $59.57 \pm 1.17 \mathrm{a}$ \\
\hline & Lower & $11.00 \pm 0.40 \mathrm{~cd}$ & $0.76 \pm 0.02 c$ & $14.54 \pm 0.85 c$ & $3.89 \pm 0.19 \mathrm{ab}$ & $8.77 \pm 0.17 b$ & $4.55 \pm 0.15 b c$ & $8.53 \pm 0.13 b$ & $11.27 \pm 0.08 c$ & $57.45 \pm 1.95 \mathrm{a}$ \\
\hline \multirow{2}{*}{ T3 } & Upper & $12.70 \pm 0.20 \mathrm{a}$ & $0.67 \pm 0.02 \mathrm{ef}$ & $19.01 \pm 0.69 \mathrm{a}$ & $4.94 \pm 0.14 \mathrm{a}$ & $8.77 \pm 0.17 b$ & $5.11 \pm 0.16 \mathrm{a}$ & $8.54 \pm 0.14 b$ & $12.78 \pm 0.34 b$ & $54.56 \pm 1.78 \mathrm{~b}$ \\
\hline & Lower & $11.50 \pm 0.50 \mathrm{bc}$ & $0.76 \pm 0.02 c$ & $15.20 \pm 0.89 b c$ & $4.14 \pm 0.14 \mathrm{ab}$ & $7.64 \pm 0.14 \mathrm{e}$ & $4.64 \pm 0.10 \mathrm{bc}$ & $7.50 \pm 0.20 \mathrm{e}$ & $9.91 \pm 0.49 \mathrm{~d}$ & $52.35 \pm 1.92 b c$ \\
\hline \multirow{2}{*}{$\mathrm{T} 4$} & Upper & $11.10 \pm 0.10 \mathrm{~cd}$ & $0.68 \pm 0.01 \mathrm{def}$ & $16.40 \pm 0.20 \mathrm{~b}$ & $4.27 \pm 0.17 \mathrm{ab}$ & $8.69 \pm 0.19 b$ & $4.69 \pm 0.19 b$ & $8.44 \pm 0.14 b$ & $12.47 \pm 0.21 b$ & $57.43 \pm 0.97 a$ \\
\hline & Lower & $10.90 \pm 0.30 \mathrm{cde}$ & $0.70 \pm 0.01 d$ & $15.49 \pm 0.57 \mathrm{bc}$ & $3.45 \pm 0.10 \mathrm{~b}$ & $8.40 \pm 0.20 c$ & $4.46 \pm 0.16 b c$ & $8.17 \pm 0.17 c$ & $11.61 \pm 0.35 c$ & $54.56 \pm 1.78 b$ \\
\hline
\end{tabular}


sucrose $(5.11 \%)$ were the highest in the upper fruits of $\mathrm{T} 3$, followed by the upper layer fruits of T2. However, Titratable acid were the highest and the contents of the soluble solids, TSS/acid ratio, reducing sugar, sugar, sucrose, total sugar, sugar/acid ratio and Vc content were the lowest in control. Therefore, it was explained that $\mathrm{T} 2$ and $\mathrm{T} 3$ were beneficial to improve the internal quality of fruit.

\subsection{The Correlation Analysis of Leaf and Soil Nutrient}

From Table 5, the contents of B, $\mathrm{Zn}$ and Fe in leaves were significantly correlated with soil nutrient. However, the contents of $\mathrm{N}, \mathrm{P}, \mathrm{K}, \mathrm{Mg}$ were no significant correlations with soil nutrient contents. There was a significant negative correlation between $\mathrm{B}$ content in leaf and $\mathrm{pH}$ value in soil, and positively correlated with available $\mathrm{B}$, organic matter, total $\mathrm{N}$, total $\mathrm{P}$, available $\mathrm{N}$, available $\mathrm{P}$, available $\mathrm{K}$, available $\mathrm{Cu}$, available $\mathrm{Zn}$, available $\mathrm{Fe}$ in soil. The $\mathrm{Mg}$ content in leaves was positively correlated with available $\mathrm{N}$ and available Fe, and negatively correlated with other indexes, especially with available $\mathrm{Cu}$, available $\mathrm{Zn}$ and $\mathrm{pH}$. While the Fe content of leaves was negatively correlated with $\mathrm{pH}$ value and exchangeable Ca. It was positively correlated with other indexes and had a significant positive correlation with total $\mathrm{K}$, available $\mathrm{K}$, available $\mathrm{P}$, available $\mathrm{B}$, available $\mathrm{Cu}$ and available $\mathrm{Zn}$. It shows that leaf nutrient and soil nutrient were closely related.

\subsection{The Correlation Analysis of Soil Nutrients and Fruit Yield and Quality}

According to Table 6, the $\mathrm{pH}$ value was negatively correlated with the fruit yield

Table 5. Correlation between leaf and soil nutrient parameters.

\begin{tabular}{|c|c|c|c|c|c|c|c|}
\hline $\mathrm{R}$ & $\mathrm{N}\left(\mathrm{g} \cdot \mathrm{kg}^{-1}\right)$ & $\mathrm{P}\left(\mathrm{g} \cdot \mathrm{kg}^{-1}\right)$ & $\mathrm{K}\left(\mathrm{g} \cdot \mathrm{kg}^{-1}\right)$ & $\mathrm{B}\left(\mathrm{mg} \cdot \mathrm{kg}^{-1}\right)$ & $\mathrm{Mg}\left(\mathrm{mg} \cdot \mathrm{kg}^{-1}\right)$ & $\mathrm{Zn}\left(\mathrm{mg} \cdot \mathrm{kg}^{-1}\right)$ & $\mathrm{Fe}\left(\mathrm{mg} \cdot \mathrm{kg}^{-1}\right)$ \\
\hline $\mathrm{pH}$ & $-0.370^{*}$ & $-0.571^{\star \star}$ & -0.153 & $-0.469^{\star *}$ & $-0.523^{\star *}$ & -0.073 & -0.025 \\
\hline Organic mater $\left(\mathrm{g} \cdot \mathrm{kg}^{-1}\right)$ & -0.080 & -0.025 & $0.398^{*}$ & $0.631^{\star *}$ & -0.144 & $0.384^{*}$ & $0.396^{*}$ \\
\hline $\mathrm{N}\left(\mathrm{g} \cdot \mathrm{kg}^{-1}\right)$ & 0.147 & 0.116 & 0.304 & $0.640^{\star *}$ & -0.073 & 0.224 & $0.398^{*}$ \\
\hline $\mathrm{P}\left(\mathrm{g} \cdot \mathrm{kg}^{-1}\right)$ & -0.318 & -0.039 & 0.096 & $0.560^{* *}$ & -0.302 & $0.477^{\star *}$ & $0.464^{* *}$ \\
\hline $\mathrm{K}\left(\mathrm{g} \cdot \mathrm{kg}^{-1}\right)$ & 0.062 & -0.018 & 0.108 & 0.06 & -0.331 & $-0.522^{\star *}$ & $0.531^{\star *}$ \\
\hline Available N (mg.kg-1) & 0.053 & 0.154 & 0.236 & $0.677^{\star *}$ & 0.113 & $0.544^{* *}$ & 0.198 \\
\hline Available P (mg.kg-1) & -0.251 & 0.060 & 0.242 & $0.745^{\star *}$ & -0.252 & $0.368^{*}$ & $0.649^{* *}$ \\
\hline Rapidly available $\mathrm{K}\left(\mathrm{mg} \cdot \mathrm{kg}^{-1}\right.$ ) & -0.024 & 0.018 & $0.416^{\star}$ & $0.732^{\star *}$ & -0.198 & $0.372^{\star}$ & $0.648^{\star *}$ \\
\hline Available Cu (mg.kg $\left.{ }^{-1}\right)$ & -0.303 & 0.005 & 0.191 & $0.630^{* *}$ & $-0.438^{*}$ & 0.209 & $0.766^{* *}$ \\
\hline Available $\mathrm{Zn}\left(\mathrm{mg} \cdot \mathrm{kg}^{-1}\right)$ & -0.028 & 0.089 & 0.357 & $0.776^{* *}$ & -0.210 & 0.236 & $0.672^{\star *}$ \\
\hline Available Fe $\left(\mathrm{mg} \cdot \mathrm{kg}^{-1}\right)$ & 0.138 & 0.227 & $0.493^{* *}$ & $0.751^{\star *}$ & 0.093 & $0.625^{\star *}$ & $0.377^{\star}$ \\
\hline Available Mn (mg.kg $\left.{ }^{-1}\right)$ & $-0.407^{\star}$ & -0.166 & -0.179 & 0.279 & -0.215 & $0.581^{\star *}$ & 0.079 \\
\hline Exchangeable Ca $\left(\mathrm{g} \cdot \mathrm{kg}^{-1}\right)$ & 0.247 & -0.210 & 0.189 & -0.257 & -0.164 & -0.303 & -0.019 \\
\hline Exchangeable $\mathrm{Mg}\left(\mathrm{g} \cdot \mathrm{kg}^{-1}\right)$ & -0.255 & -0.313 & 0.033 & 0.325 & -0.143 & $0.548^{\star *}$ & 0.079 \\
\hline
\end{tabular}


Table 6. Correlation between soil nutrient and fruit yield and quality.

\begin{tabular}{|c|c|c|c|c|c|c|c|c|c|c|c|c|c|}
\hline $\mathrm{R}$ & $\begin{array}{c}\text { Fruit } \\
\text { weight }(\mathrm{g})\end{array}$ & $\begin{array}{l}\text { Fruit } \\
\text { shape } \\
\text { index }\end{array}$ & $\begin{array}{l}\text { Fruit peel } \\
\text { thickness } \\
(\mathrm{mm})\end{array}$ & $\begin{array}{c}\text { Juice rate } \\
(\%)\end{array}$ & $\begin{array}{l}\text { Edible } \\
\text { rate (\%) }\end{array}$ & TSS (\%) & TA (\%) & $\begin{array}{l}\text { TSS/ } \\
\text { acid }\end{array}$ & $\begin{array}{c}\text { Total } \\
\text { sugar } \\
(\%)\end{array}$ & $\begin{array}{l}\text { Sugar/ } \\
\text { acid }\end{array}$ & $\begin{array}{c}\mathrm{Vc} \\
\text { content } \\
\left(\mathrm{mg} \cdot \mathrm{kg}^{-1}\right)\end{array}$ & $\begin{array}{c}\text { Fruit } \\
\text { number } \\
\text { n plant }\end{array}$ & $\begin{array}{c}\text { Fruit } \\
\text { yield }(\mathrm{kg} \\
\left.\text { plant }^{-1}\right)\end{array}$ \\
\hline $\mathrm{pH}$ & -0.150 & -0.265 & -0.101 & -0.063 & 0.015 & 0.065 & -0.098 & 0.145 & -0.107 & 0.040 & -0.151 & -0.294 & $-0.364^{*}$ \\
\hline $\begin{array}{c}\text { Organic } \\
\text { mater }\left(\mathrm{g} \cdot \mathrm{kg}^{-1}\right)\end{array}$ & $0.507^{\star *}$ & $0.491^{* *}$ & $0.708^{\star *}$ & $-0.847^{* *}$ & $-0.630^{\star *}$ & $0.758^{\star *}$ & $-0.864^{\star *}$ & $0.849^{* *}$ & $0.743^{* *}$ & $0.831^{\star *}$ & $0.501^{* *}$ & $0.788^{\star *}$ & $0.686^{* *}$ \\
\hline $\mathrm{N}\left(\mathrm{g} \cdot \mathrm{kg}^{-1}\right)$ & $0.681^{\star \star}$ & $0.632^{\star *}$ & $0.856^{\star *}$ & $-0.716^{\star \star}$ & $-0.679^{* *}$ & $0.723^{\star *}$ & $-0.925^{\star \star}$ & $0.836^{* *}$ & $0.793^{* *}$ & $0.859^{\star *}$ & $0.688^{\star *}$ & $0.746^{\star *}$ & $0.767^{* *}$ \\
\hline $\mathrm{P}\left(\mathrm{g} \cdot \mathrm{kg}^{-1}\right)$ & $0.387^{\star}$ & 0.323 & $0.485^{\star *}$ & $-0.830^{* *}$ & $-0.523^{\star *}$ & $0.838^{* *}$ & $-0.709^{\star *}$ & $0.845^{\star *}$ & $0.746^{* *}$ & $0.785^{\star *}$ & $0.447^{\star}$ & $0.635^{\star *}$ & $0.505^{* *}$ \\
\hline $\mathrm{K}\left(\mathrm{g} \cdot \mathrm{kg}^{-1}\right)$ & $0.476^{\star *}$ & -0.204 & 0.221 & 0.062 & -0.163 & -0.150 & -0.202 & 0.031 & 0.245 & 0.259 & 0.342 & -0.253 & 0.137 \\
\hline $\begin{array}{c}\text { Available N } \\
\left(\mathrm{mg} \cdot \mathrm{kg}^{-1}\right)\end{array}$ & $0.447^{*}$ & $0.658^{* *}$ & $0.618^{\star *}$ & $-0.707^{\star *}$ & $-0.534^{\star *}$ & $0.798^{\star *}$ & $-0.764^{\star *}$ & $0.811^{* *}$ & $0.754^{* *}$ & $0.766^{* *}$ & $0.452^{*}$ & $0.899^{* *}$ & $0.718^{* *}$ \\
\hline $\begin{array}{c}\text { Available P } \\
\left(\mathrm{mg} \cdot \mathrm{kg}^{-1}\right)\end{array}$ & $0.567^{\star *}$ & $0.451^{*}$ & $0.600^{\star *}$ & $-0.829^{\star \star}$ & $-0.566^{\star *}$ & $0.781^{\star *}$ & $-0.713^{\star \star}$ & $0.803^{* *}$ & $0.824^{* *}$ & $0.826^{* *}$ & $0.590^{* *}$ & $0.671^{* *}$ & $0.656^{* *}$ \\
\hline $\begin{array}{c}\text { Rapidly } \\
\text { available K } \\
\left(\mathrm{mg} \cdot \mathrm{kg}^{-1}\right)\end{array}$ & $0.742^{* *}$ & $0.567^{* *}$ & $0.793^{* *}$ & $-0.872^{\star *}$ & $-0.775^{\star *}$ & $0.772^{* *}$ & $-0.853^{\star *}$ & $0.862^{* *}$ & $0.896^{\star *}$ & $0.925^{* *}$ & $0.671^{* *}$ & $0.760^{* *}$ & $0.774^{* *}$ \\
\hline $\begin{array}{c}\text { Available B } \\
\left(\mathrm{mg} \cdot \mathrm{kg}^{-1}\right)\end{array}$ & $0.818^{* *}$ & 0.237 & $0.570^{* *}$ & -0.219 & $-0.454^{\star}$ & -0.009 & $-0.429^{\star}$ & 0.213 & $0.538^{\star *}$ & $0.527^{\star *}$ & $0.673^{* *}$ & 0.045 & 0.219 \\
\hline $\begin{array}{l}\text { Available } \mathrm{Cu} \\
\left(\mathrm{mg} \cdot \mathrm{kg}^{-1}\right)\end{array}$ & $0.617^{* *}$ & 0.245 & $0.554^{\star *}$ & $-0.793^{\star *}$ & $-0.596^{* *}$ & $0.691^{* *}$ & $-0.704^{\star *}$ & $0.764^{* *}$ & $0.841^{* *}$ & $0.850^{\star *}$ & $0.630^{* *}$ & $0.473^{* *}$ & $0.490^{* *}$ \\
\hline $\begin{array}{l}\text { Available } \mathrm{Zn} \\
\left(\mathrm{mg} \cdot \mathrm{kg}^{-1}\right)\end{array}$ & $0.785^{* *}$ & $0.572^{* *}$ & $0.825^{\star *}$ & $-0.810^{\star *}$ & $-0.714^{* *}$ & $0.745^{\star *}$ & $-0.894^{\star *}$ & $0.856^{* *}$ & $0.938^{* *}$ & $0.957^{\star *}$ & $0.746^{* *}$ & $0.743^{* *}$ & $0.811^{* *}$ \\
\hline $\begin{array}{l}\text { Available Fe } \\
\left(\mathrm{mg} \cdot \mathrm{kg}^{-1}\right)\end{array}$ & $0.559^{* *}$ & $0.805^{* *}$ & $0.781^{* *}$ & $-0.893^{\star *}$ & $-0.664^{\star *}$ & $0.871^{\star *}$ & $-0.748^{\star *}$ & $0.857^{\star *}$ & $0.756^{* *}$ & $0.780^{* *}$ & $0.569^{* *}$ & $0.824^{* *}$ & $0.781^{* *}$ \\
\hline $\begin{array}{l}\text { Available Mn } \\
\left(\mathrm{mg} \cdot \mathrm{kg}^{-1}\right)\end{array}$ & -0.009 & 0.306 & 0.260 & $-0.636^{\star *}$ & -0.207 & $0.826^{\star *}$ & $-0.484^{\star *}$ & $0.720^{* *}$ & $0.400^{*}$ & $0.469^{* *}$ & 0.153 & $0.526^{* *}$ & $0.621^{* *}$ \\
\hline $\begin{array}{c}\text { Exchangeable } \\
\mathrm{Ca}\left(\mathrm{g} \cdot \mathrm{kg}^{-1}\right)\end{array}$ & 0.223 & -0.029 & 0.286 & 0.046 & -0.184 & -0.126 & -0.277 & 0.070 & 0.028 & 0.145 & 0.104 & -0.120 & 0.201 \\
\hline $\begin{array}{l}\text { Exchangeable } \\
\mathrm{Mg}\left(\mathrm{g} \cdot \mathrm{kg}^{-1}\right)\end{array}$ & 0.174 & $0.560^{* *}$ & $0.474^{\star *}$ & $-0.633^{\star *}$ & -0.331 & $0.819^{* *}$ & $-0.634^{* *}$ & $0.781^{* *}$ & $0.448^{*}$ & $0.555^{\star *}$ & 0.214 & $0.713^{\star *}$ & 0.019 \\
\hline
\end{tabular}

Note: Correlation is significant at the 0.01 level. Correlation is significant at the 0.05 level, The same as followed.

and quality index, but only the correlation between $\mathrm{pH}$ value and yield per plant reached significant level. There was a significant positive correlation between total $\mathrm{K}$ and fruit weight, and the other indexes did not reach significant level. The contents of organic matter, total $\mathrm{N}$, total $\mathrm{P}$, available $\mathrm{N}$, available $\mathrm{P}$, available $\mathrm{K}$, available $\mathrm{B}$, available $\mathrm{Cu}$, available $\mathrm{Zn}$, available $\mathrm{Fe}$, available $\mathrm{Mn}$ and exchangeable $\mathrm{Mg}$ were negative correlations with the juice yield, edible rate and titratable acid. There was a significant positive correlation with fruit weight, fruit thickness, total sugar, solid acid ratio, sugar and acid ratio, Vc content and fruit yield. There was a significant positive correlation between available B content, fruit weight, fruit thickness, total sugar, sugar and acid ratio and Vc content, and negatively correlated with juice yield, edible rate and titratable acid. The effective 
Fe and $\mathrm{Zn}$ content was negatively correlated with the juice yield, edible rate and titratable acid, which was significantly correlated with other fruit yield and quality indexes. Therefore, soil nutrients have played a very important role in improving fruit quality.

\subsection{The Correlation Analysis of Leaf Nutrients and Fruit Yield and Quality}

According to Table 7, the correlation between $\mathrm{K}, \mathrm{B}, \mathrm{Zn}, \mathrm{Fe}$ and fruit yield and quality index was significant, while the $\mathrm{N}, \mathrm{P}, \mathrm{Mg}$ were not significant correlations with the yield and quality of fruit. There was a significant positive correlation between the $\mathrm{K}$ content, fruit weight, fruit thickness, fruit number, fruit yield, total sugar, the ratio of sugar to acid, and the content of $\mathrm{Vc}$, and negatively correlated with juice yield and edible rate. Negative correlations were shown between the $\mathrm{B}$ content and juice rate, edible rate and titratable acidity. The content of B was a significant positive correlation with the yield and quality of other indexes. There was a significant negative correlation between Fe content and juice yield and edible rate, and negatively correlated with titratable acid. There was a significant positive correlation with single fruit weight, single yield, fruit thickness, total sugar, and the solid acid ratio was significantly positive correlation. The content of $\mathrm{Zn}$ was significant negatively with the juice rate, and was negatively correlated with the edible rate, and was positively correlated with the soluble solids, fruit shape index, fruit number per plant and yield per plant. It was found that the leaf nutrient contents were negatively correlated with juice rate and edible rate.

\section{Discussion}

\subsection{Effects of Fertilization on Nutrient Content and Correlations}

Different fertilization treatments had a significant effect on soil nutrient content,

Table 7. Correlation between leaf nutrient and fruit yield and quality.

\begin{tabular}{|c|c|c|c|c|c|c|c|c|c|c|c|c|c|}
\hline $\mathrm{R}$ & $\begin{array}{c}\text { Fruit } \\
\text { weight (g) }\end{array}$ & $\begin{array}{l}\text { Fruit } \\
\text { shape } \\
\text { index }\end{array}$ & $\begin{array}{l}\text { Fruit peel } \\
\text { thickness } \\
\quad(\mathrm{mm})\end{array}$ & $\begin{array}{l}\text { Juice rate } \\
\text { (\%) }\end{array}$ & $\begin{array}{c}\text { Edible } \\
\text { rate }(\%)\end{array}$ & $\begin{array}{l}\text { TSS } \\
(\%)\end{array}$ & TA (\%) & $\begin{array}{l}\text { TSS/ } \\
\text { acid }\end{array}$ & $\begin{array}{c}\text { Total } \\
\text { sugar (\%) }\end{array}$ & $\begin{array}{l}\text { Sugar/ } \\
\text { acid }\end{array}$ & $\begin{array}{c}\mathrm{Vc} \\
\text { content } \\
\left(\mathrm{mg} \cdot \mathrm{kg}^{-1}\right)\end{array}$ & $\begin{array}{c}\text { Fruit } \\
\text { number } \\
\text { n plant }\end{array}$ & $\begin{array}{c}\text { Fruit } \\
\text { yield }(\mathrm{kg} \\
\left.\text { plant }^{-1}\right)\end{array}$ \\
\hline $\mathrm{N}\left(\mathrm{g} \cdot \mathrm{kg}^{-1}\right)$ & 0.285 & $0.381^{\star}$ & 0.310 & -0.117 & $-0.432^{*}$ & -0.104 & 0.085 & -0.145 & 0.087 & -0.035 & 0.085 & 0.309 & $0.472^{\star *}$ \\
\hline $\mathrm{P}\left(\mathrm{g} \cdot \mathrm{kg}^{-1}\right)$ & 0.274 & 0.290 & 0.191 & -0.269 & $-0.375^{\star}$ & 0.057 & 0.089 & -0.042 & 0.273 & 0.088 & 0.203 & 0.29 & $0.529^{\star *}$ \\
\hline $\mathrm{K}\left(\mathrm{g} \cdot \mathrm{kg}^{-1}\right)$ & $0.568^{\star *}$ & $0.554^{\star \star}$ & $0.511^{\star *}$ & $-0.525^{\star *}$ & $-0.679^{\star *}$ & 0.142 & -0.254 & 0.196 & $0.451^{\star}$ & $0.371^{\star}$ & $0.380^{*}$ & $0.504^{\star *}$ & $0.727^{\star *}$ \\
\hline $\mathrm{B}\left(\mathrm{mg} \cdot \mathrm{kg}^{-1}\right)$ & $0.760^{* *}$ & $0.728^{\star *}$ & $0.727^{\star *}$ & $-0.782^{\star *}$ & $-0.758^{\star *}$ & $0.589^{* *}$ & $-0.555^{\star *}$ & $0.586^{* *}$ & $0.833^{\star *}$ & $0.725^{\star *}$ & $0.693^{* *}$ & $0.761^{\star *}$ & $0.940^{* *}$ \\
\hline $\begin{array}{c}\mathrm{Mg} \\
\left(\mathrm{mg} \cdot \mathrm{kg}^{-1}\right)\end{array}$ & -0.094 & 0.343 & 0.001 & -0.005 & -0.136 & -0.089 & 0.286 & -0.238 & -0.135 & -0.264 & -0.221 & 0.358 & 0.306 \\
\hline $\begin{array}{c}\mathrm{Zn} \\
\left(\mathrm{mg} \cdot \mathrm{kg}^{-1}\right)\end{array}$ & 0.024 & $0.486^{* *}$ & 0.264 & $-0.621^{\star *}$ & $-0.449^{*}$ & $0.596^{\star *}$ & -0.185 & $0.416^{\star}$ & 0.272 & 0.227 & -0.030 & $0.689^{\star *}$ & $0.453^{*}$ \\
\hline $\begin{array}{c}\mathrm{Fe} \\
\left(\mathrm{mg} \cdot \mathrm{kg}^{-1}\right)\end{array}$ & $0.842^{* *}$ & 0.269 & $0.585^{\star *}$ & $-0.533^{\star *}$ & $-0.593^{\star *}$ & 0.325 & $-0.454^{*}$ & $0.426^{*}$ & $0.730^{\star *}$ & $0.668^{* *}$ & $0.789^{\star *}$ & 0.171 & $0.555^{\star *}$ \\
\hline
\end{tabular}


and different fertilization treatments had significant difference, but the evaluation results of fertilization treatments were different in the existing reports. Some researchers suggested that organic fertilizer alone significantly promote the absorption of calcium, and promote the transformation of nitrate nitrogen, adjust the soil nitrate content changes [18]. It was also suggested that organic manure could increase soil organic matter, total $\mathrm{N}$, available $\mathrm{P}$, available $\mathrm{K}$, exchangeable $\mathrm{Ca}$, exchangeable $\mathrm{Mg}, \mathrm{pH}$ value and soil fertility [19]. As well as the researchers suggested that the orchard increased organic fertilizer can significantly improve the nutrient contents of leaf [20] [21]. More studies have found that the rational application of chemical fertilizers and organic fertilizers can significantly improve the soil fertility and fruit yield [22] [23].

In this experiment, the results showed that the nutrient contents in soil increased with the increase of organic manure application. The nutrient contents in T2 and T3 were significantly increased, and the soil nutrient contents of 0 - 20 $\mathrm{cm}$ depth in $\mathrm{T} 2$ were the highest, which indicated that the increase of organic fertilizers was beneficial to improve soil available nutrient, and organic fertilizers with chemical fertilizers were better (T2). Furthermore, the nutrient contents of vegetative leaves in $\mathrm{T} 2$ were also the highest. It indicated that most of the nutrients in the tree came from the soil, and the leaf nutrient was closely related to the soil nutrient [24]. In addition, the results showed that the Mg content in the leaves was positively correlated with the available $\mathrm{N}$ and available $\mathrm{Fe}$, and negatively correlated with other parameters, which indicated that the Mg deficiency in leaves was not only caused by the corresponding elements in the soil, and applying single fertilizer could not meet the tree's requirement for nutrient [25]. The results also showed that there was a positive correlation between Fe, B content in leaves and soil nutrient content, it indicated that soil nutrients directly affect the Fe and B nutrient content in leaves, and fertilization could significantly improve to the nutrient level in leaves. There were many factors that affect the relationship between soil and leaf nutrient, but there has not yet reached a uniform conclusion about the correlation in the existing reports. Thus the specific relationship and the reasons need to be further studied.

\subsection{Effects of Fertilization on Fruit Yield and Quality and Correlations}

The nutrient of leaf and soil had significant effect on fruit yield and quality, especially the index of leaves' nutrient content can respond to the nutritional requirements more accurately for optimum fruit yield [4]. Zang et al. suggested that the application of organic fertilizer can increase the soluble solids, Vc content and solid acid ratio, reduce the content of titratable acid [26]. Wang et al. suggested that the application of different ratio of compound fertilizer is enable to improve the yield and quality of citrus [27]. Marathe reported that the application of FYM (to supply $50 \% \mathrm{~N})+50 \% \mathrm{RDF}\left(1000 \mathrm{~g} \mathrm{~N}+400 \mathrm{~g} \mathrm{P}_{2} \mathrm{O}_{5}+400 \mathrm{~g}\right.$ $\mathrm{K}_{2} \mathrm{O}$ tree $^{-1}$ year $^{-1}$ ) was optimum for sustaining higher fruit yield and quality of sweet orange [28]. In this study, the fruit and yield were the highest, the fruit 
peel was thicker, and the content of sugar, total sugar, $\mathrm{Vc}$ and rate of sugar and acid were the highest in $\mathrm{T} 2$, it indicated that $\mathrm{T} 2$ can improve the ability of anti-fungal invasion and enhance the storability [29].

The relationship between soil or leaf nutrient content and fruit yield and quality was complex. Zhang et al. found that available $\mathrm{P}$ in soil had the greatest effects on fruit quality, followed by $\mathrm{K}, \mathrm{N}$, and $\mathrm{P}$ in leaf could significantly increase the content of $\mathrm{Vc}$ and sugar, but reduce acidity, while $\mathrm{Mg}$ and $\mathrm{B}$ increase the acidity of fruit [30]. In addition, researchers have suggested that the content of soluble solids in fruit was negatively correlated with soil $\mathrm{pH}$ value, and there was a significant positive correlation with soil organic matter, available $\mathrm{N}, \mathrm{Fe}, \mathrm{Mn}$ and $\mathrm{Zn}$. The content of $\mathrm{Vc}$ in fruit was positively correlated with soil $\mathrm{pH}$ and available $\mathrm{K}$, and negatively correlated with soil organic matter and other nutrient contents [4]. It has also been suggested that most of the nutrients in citrus leaves were negatively correlated with the soluble solids of fruit quality, and positively correlated with Vc content [31]. In this study, we found that the content of available $\mathrm{K}, \mathrm{B}, \mathrm{Fe}$ and $\mathrm{Zn}$ in soil increased the content of mineral elements in leaves, which increased fruit peel thickness, fruit weight, total sugar and Vc content etc. The similar results have been reported by Aula et al. [32], Khayyat et al. [33], Patel et al. [34] and Asadi et al. [35]. Meanwhile, B, Fe, Zn content and titratable acid were significantly negatively correlated, it indicated that it could increase sugar and reduce acid content with increasing B, Fe, Zn content, Furthermore, the content of $\mathrm{B}$ and $\mathrm{Zn}$ was positively correlated with the soluble solid, and the content of available $\mathrm{B}$ and $\mathrm{Zn}$ was helpful to increase the soluble solids content of fruit. Therefore, foliage spraying $\mathrm{K}, \mathrm{B}, \mathrm{Fe}, \mathrm{Zn}$ appropriately is the effective way for improving fruit yield and quality in the orchard fertilization practice.

\section{References}

[1] Huang, C.H., Qu, X.Y., Liu, K. P., Leng, J.H., Tu, G.Q., Li, B.M., et al. (2014) Analysis of Soil Physicochemical Properties, Leaf Nutrients and Fruit Qualities in the Orchards of "Jinkui" Kiwifruit (Actinidiadeliciosa). Journal of Fruit Science, 31, 1091-1099.

[2] Yu, N.W., Li, J.C., Wang, J.Z., Cai, Z.M., Sha, S.F. and Li, H.J. (2013) Investigation and Analysis on Nutrient Status of Soil and Leaves in "Nan guoli" Pear Orchards in Liaoning Province. Journal of Fruit Science, 30, 254-259.

[3] Dinnes, D.L., Karlen, D.L., Jaynes, D.B., Kaspar, T.K., Hatfield, J.L., Colvin, T.S., et al. (2002) Nitrogen Management Strategies to Reduce Nitrate Leaching in Tiledrained Midwestern Soils. Agronomy Journal, 94, 153-171. https://doi.org/10.2134/agronj2002.0153

[4] Yang, S.Q. (2008) Effects of Soil and Leaf Nutrient Status on Yield and Quality of Citrus. Southwest University, Beibei.

[5] Qiu, X.K., Dong, Y.J., Wan, Y.S., Hu, G.Q. and Wang, Y.H. (2010) Effects of Different Fertilizing Treatments on Contents of Soil Nutrients and Soil Enzyme Activity. Soils, 42, 249-255.

[6] Ling, L.L., Peng, L.Z., Chun, C.P., Jiang, C.L. and Cao, L. (2012) The Relationship between Leaf Nutrients and Fruit Quality of Navel Orange in Southern Jiangxi 
Province of China. Plant Nutrition and Fertilizer Science, 18, 947-954.

[7] Thamrin, M., Susanto, S., Susila, A.D. and Dan, A.S. (2014). Correlation between Nitrogen, Phosphorus and Potassium Leaf Nutrient with Fruit Production of Pummelo Citrus (citrus maxima). Asian Journal of Applied Sciences, 7, 129-139. https://doi.org/10.3923/ajaps.2014.129.139

[8] Lu, C., Xue, X.M., Wang, C.L., An, G.N. and Wang, J.Z. (2011) Correlation Analysis Oil Fruit Quality and Leaves Nutrition and Soft Nutrient in Apple Orchard of Shandong Province. Chinese Agricultural Science Bulletin, 27, 168-172.

[9] Song, X.H., Xie, K., Zhao, H.B., Li, Y.L., Dong, C.X., Xu, Y.C., et al. (2012) Effects of Different Organic Fertilizers on Tree Growth, Yield, Fruit Quality, and Soil Microorganisms in a Pear Orchard. European Journal of Horticultural Science, 77, 204210.

[10] Liu, Z.K. (2010) Early Knot and High-Yielding Cultivation Techniques of Citrus Grandis Var. longanyou. Chinese Fruit Trees in Southern China, 39, 64-65.

[11] Xiong, A.M. (2012) High-quality Fruit Production Technology of Citrus grandis var. longanyou. Chinese fruit trees in Southern China, 41, 83.

[12] Tang, Y.Q., Peng, L.Z., Chun, C.P., Ling, L.L., Fang, Y.W. and Yan, X. (2013) Correlation Analysis on Nutrient Element Contents in Orchard Soils and Sweet Orange Leaves in Southern Jiangxi Province of China. ActaHorticulturaeSinica, 40,623-632.

[13] Zhang, C.M. (2013) The Effects of Different Fertilizer Treatments on the Soil and Leaf Nutrient and the Metbolism of Citric Acid of Navel in Zigui. Wuhan: Huazhong Agricultural University.

[14] Bao, S.D. (2000) Soil Agrochemical Analysis.3rd Edition, China Agricultural Publishing Press, Beijing.

[15] Mu, H.T.R.Z., Wu, Z.B., Gu, L. M.R.K. and Shi, Y.J. (2015) Effects of Different Proportions of Organic-inorganic Fertilizers on Soil Nutrition, Photosynthetic Parameters and Leaf-Fruit Ratio of Junzao. Chinese Journal of Soil Science, 46, 405-411.

[16] Zhuang, Y.M., Wang, R.J., Chen, L.X., Xie, Z.N., Xu, W.B., Huang, Y.Z., et al. (1991) Optimum Range of Mineral Element Contents in the Leaves of Guanxi Honey Pomelo (Citrus grandis). Journal of Fujian Academy of Agriculture Sciences, 6, 52-58.

[17] Diao, L.H. (2013) Seasonal Changes of Nutrient Element Contents in Leaves of Four Citrus Cultivars. Beibei: Southwest University.

[18] Hu, L.P., Xie, T.Z., Zou, Y.L., Wang, T.P., Chen, Q. and Ye, X.Y. (2011) Effects of Different Fertilizations on the Calcium Content in the Apple Fruits and Soil Nutrient. Northern horticulture, 14, 16-19.

[19] Zang, X.P., Zhou, Z.X., Lin, X.E., Dai, M.J., Ge, Y., Liu, Y.X., et al. (2016) Effects of different Organic Manure Application Rate on Mango Fruit Quality and Soil Fertility. Chinese Soil and Fertilizer, 1, 98-101.

[20] Chu, C.B., Wu, S.H., Zhang, X.Y., Zhou, D.P., Fan, J.Q. and Jiang, Z.F. (2012) Effects of Organic Fertilizer Application on Soil Fertility and Leaf Nutrients and Fruit Quality of Citrus. Acta Agriculturae Shanghai, 28, 65-68.

[21] Wang, Y.X. (2015) Effects of Organic Fertilizerson Soil Nutrient, Tree Nutrition and Fruit Quality of Feicheng Peach. Shandong Agricultural University, Tai'an.

[22] Luo, H., Li, M., Hu, D.G., Song, H.R., Hao, Y.J. and Zhang, L.Z. (2012) Effects of Organic Fertilization on Fruit Yield and Quality of Feicheng peach ( Prunuspersica cv. Feicheng). Plant Nutrition and Fertilizer Science, 18, 955-964.

[23] Zhao, Z.P., Tong, Y. A., Liu, F. and Wang, X.Y. (2013) Effects of Different Long-Term Fertilization Patterns on Fuji Apple Yield, Quality, and Soil Fertility on 
Weibei Dryland. Chinese Journal of Applied Ecology, 24, 3091-3098.

[24] Zhao, Z.G., Tang, F.L., Li, S.H., Huang, Z.Q., Shi, Y.P., Fu, C.M., et al. (2013) Corrlation Analysis on Nutrition Status of Soil and Leaf Nutrients for Pyruspyrifolia cv. Hosui Citrus demonstration Orchard. Guihaia, 33, 171-176.

[25] Wei, X.M., Liao, M.A., Zhou, Y.G. and Dai, S.Y. (2008) Study on the Correlation between Leaf Nutrition and Soil Nutrient of PyruspyrifoliaNak. cv. Jinhua. Xiandai Horticulture, 9, 4-6.

[26] Zang, X.P., Lin, X.E., Zhou, Z.X., Tan, L., Ge, Y., Dai, M.J., et al. (2015) Effects of Different Fertilization Treatments on Quality of Mango Fruit and Soil Fertility. Subtropical Plant Science, 44, 146-149.

[27] Wang, R., Wei, Y.Z., Yang, X.E., Tang, C.H., Jia, Y.B., Hong, C.L., et al. (2004) Effects of Different Proportioning Compound Fertilizers on Yield and Quality of Citrus. Journal of Zhejiang Agricultural Sciences, 1, 243-245.

[28] Marathe, R.A. (2005) Effect Of Integrated Nutrient Management On Soil Properties, Yield and Quality Of Sweet Orange (Citrus Sinensis Osbeck) Grown on Vertisol.

[29] He, T.F. (1999) Chinese Pomelo Cultivation. China Agricultural Publishing Press, Beijing, 129-137.

[30] Zhang, L., Shi, X.G., Fan, G.E. and Lin, M. (2010) Relationship between Soil and Leaf Nutrient Quality and Fruit Quality of Satsuma Mandarine. Journal of Zhejiang Agricultural Sciences, 1, 961-963.

[31] Xu, H.Y., Xiong, W., Yang, C.F., Huang, Y. and Zeng, M. (2012) Correlation Analysis of Mineral Nutrients in Citrus Leaf and Fruit Quality in Kaixian County. Journal of Southwest University (Natural Science Edition), 34, 27-32.

[32] Aular, J., Rengel, M., Montaño, M. and Aular-Rodriguéz, J. (2010) Relationship between Soil and Leaf Potassium Content and "Valencia" Orange Fruit Quality. ActaHorticulturae, 868, 401-404. https://doi.org/10.17660/ActaHortic.2010.868.54

[33] Khayyat, M., Tafazoli, E., Eshghi, S. and Rajaee, S. (2007) Effect of Nitrogen, Boron, Potassium and Zinc Sprays on Yield and Fruit Quality of Date Palm. American-Eurasian Journal of Agricultural \& Environmental Sciences, 2, 289-296.

[34] Patel, P.C., Kalyansundram, N.K. and Patel, M.S. (1998) Effect of Iron Nutrition on the Yield, Chlorosis, Fruit Quality and Leaf Composition of Acidlime Trees Grown on Loamy Sand Soils. Gujarat Agricutural University Research Journal.

[35] Asadi, K.S.A., Negin, A.A. and Bizhan, M. (2007) Effect of Rates and Methods of Zinc Application on Yield and Fruit Quality of Satsuma Mandarin. Iranian Journal of Soil and Waters Sciences, 21, 1-14. 
Submit or recommend next manuscript to SCIRP and we will provide best service for you:

Accepting pre-submission inquiries through Email, Facebook, LinkedIn, Twitter, etc. A wide selection of journals (inclusive of 9 subjects, more than 200 journals)

Providing 24-hour high-quality service

User-friendly online submission system

Fair and swift peer-review system

Efficient typesetting and proofreading procedure

Display of the result of downloads and visits, as well as the number of cited articles Maximum dissemination of your research work

Submit your manuscript at: http://papersubmission.scirp.org/

Or contact wjet@scirp.org 